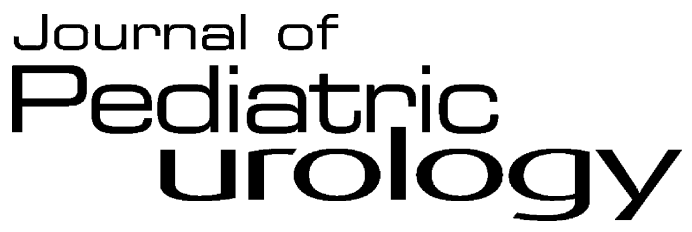

\title{
Evolving management of adolescent varicocele
}

\section{Marco Castagnetti* ${ }^{*}$ Marcello Cimador, Pieralba Catalano, MariaRita DiPace, Maria Sergio, Enrico De Grazia}

\author{
Department of Paediatric Surgery, University of Palermo, "Dipartimento Universitario Materno Infantile", \\ Via Ugo Betti 46, 90147 Palermo, Italy
}

Received 24 September 2007; accepted 31 October 2007

Available online 21 February 2008

\section{KEYWORDS \\ Varicocele; \\ Laparoscopy; \\ Recurrence; \\ Hydrocele; \\ Colour Doppler \\ ultrasound}

\begin{abstract}
Objective: To review the evolution in indications for treatment and treatment modalities for adolescent varicocele at our centre, and evaluate the impact of varicocelectomy on final outcome.

Patients and methods: Between 1995 and 2006, we treated 242 left varicoceles. Preoperative assessment included clinical evaluation, measurement of testicular volumes, and colourDoppler ultrasound (CDUS). A subinguinal varicocelectomy was performed in 124 patients (group A), and a laparoscopic non-artery-sparing Palomo procedure in the remaining 118 (group B). In group B patients, CDUS was also used to investigate the functional anatomy of varicocele, and all the veins found to be refluxing were divided during surgery. The two groups were compared with regard to indications for surgery and outcome.

Results: Over time the proportion of patients operated on because of testicular growth retardation increased. Persistence/recurrence rate was comparable between the two groups. In $13 \%$ of group $B$ patients, the deferential vein was found to be refluxing on preoperative CDUS and was divided at surgery. Hydrocele rate was higher in group A, unless the vaginalis was excised and everted during varicocelectomy. About $75 \%$ of patients with preoperative left testicular growth failure experienced postoperative catch-up growth, irrespective of treatment. Conclusion: Indications for treatment are still evolving. Varicocele can successfully be treated in the majority of cases by either a laparoscopic or subinguinal approach. Both techniques require care, and CDUS can aid in the decision making. Most patients with preoperative testicular growth failure experience postoperative catch-up growth.

(c) 2007 Journal of Pediatric Urology Company. Published by Elsevier Ltd. All rights reserved.
\end{abstract}

* Corresponding author. Tel.: +39 340292 1693; fax: +39 010 390622.

E-mail address: marcocastagnetti@hotmail.com (M. Castagnetti).

\section{Introduction}

With a prevalence as high as $40 \%$ in infertile men, varicocele is considered the main correctable cause of male infertility [1]. In children, the prevalence of varicocele increases with age from $<1 \%$ in boys aged $<10$ years 
to $15 \%$, the same prevalence as in the general adult population, after puberty $[2,3]$.

Evidence supports the principle that varicocele is associated with progressive testicular damage and that early treatment allows for improved testicular function $[4,5]$. Nevertheless, since semen analysis is not reliable before the age of 18 years, the physician has to rely upon predictors of testicular damage to select cases for surgery. Major indications for treatment usually adopted in paediatric cases include presence of a large varicocele, symptoms and ipsilateral reduced testicular size (growth failure). Only the latter can be considered a certain, yet indirect, sign of ongoing varicocele-related testicular damage [6].

If treatment is elected, the varicocele can be repaired surgically or by interventional radiology. The former may be performed at different levels, including retroperitoneal, inguinal and subinguinal, and by open surgery, with or without the aid of optical magnification, or by laparoscopy. Radiological treatment, instead, is accomplished by percutaneous embolization of the internal spermatic vein in either a retrograde or antegrade fashion.

All these methods have been reported to be associated with some risk of varicocele persistence/recurrence or postoperative hydrocele formation, the two major complications of varicocele surgery. The latter is due to iatrogenic disruption of the lymphatic system draining the testicle. Varicocele persistence/recurrence is due to collateral refluxing veins not interrupted at surgery. Indeed, in some $75 \%$ of cases, the varicocele is caused by a reflux in a single internal spermatic vein (ISV) [7] and can be treated successfully by any approach. In the remaining cases, multiple refluxing venous systems may be present. Phlebography has been considered for many years the tool of choice to investigate the vascular anatomy of varicocele [7]. More recently, we have described the use of colour-Doppler ultrasound (CDUS) for this purpose [8]. This technique has enabled us to tailor the type of treatment to each individual patient $[9,10]$.

The present study was set up to review the evolution in indications for treatment and treatment modalities at our centre, and evaluate the impact of the latter on the final success of treatment.

\section{Patients and methods}

A retrospective study was performed of all cases of varicocele operated on at our institution from January 1995 to December 2006. We identified 242 patients with a median age of 12.6 (7.1 to 16 ) years. In all, the varicocele was left sided.

Preoperatively, varicoceles were graded clinically according to Dubin and Amelar [11] Most of the patients underwent also a preoperative CDUS assessment, and varicoceles were graded haemodynamically, from grade 1 to 5 , according to Hussein [12].

Testicular volumes were usually measured using ultrasound (US), but in 19 (8\%) patients at the beginning of our experience a Prader orchidometer was used.

For the purpose of this study, the indication for surgery was classified as presence of ispilateral testicular growth failure, pain or a large varicocele. The latter was defined as either a varicocele of clinical grade 3 or haemodynamic grade 5. Testicular growth failure was defined as a size discrepancy greater than $2 \mathrm{ml}$ or more than $20 \%$ between the two sides.

Patients were divided into two groups. Group A included 124 patients (average age $12.4 \pm 3.6$ years) undergoing a subinguinal varicocelectomy between 1995 and 2001. Group B included 118 patients (average age $11.6 \pm 4.6$ years) undergoing a laparoscopic non-artery-sparing Palomo procedure between 2001 and 2006.

In group $A$, the subinguinal varicocelectomy was performed using loupes (3.5× magnification) but without the operating microscope. The testicle was always delivered during the procedure The vaginalis was left untouched in 41 (33\%) patients, whereas in the remaining $83(67 \%)$ it was opened by partial excision with or without eversion as for hydrocelectomy [13].

In all group B patients, a comprehensive preoperative evaluation of the vascular anatomy of the varicocele was performed by CDUS, as previously described [8]. Briefly, with the patients at rest and during Valsalva manoeuvre both the inguinal canal and the left iliac fossa were examined, in order to detect any possible retrograde blood flow in the ISV, cremasteric vein(s) and deferential vein(s). The latter can be visualized when dilated as a vessel running in the left iliac fossa over the external iliac vessels. A reflux is visualized on spectral analysis as a rise of signal above the baseline lasting more than $2 \mathrm{~s}$ (Fig. 1).

Based on CDUS findings, group B patients presenting a reflux in the ISV only were treated by a standard nonartery-sparing laparoscopic Palomo procedure, whereas in the others all the vessels found to be refluxing were coagulated and divided. In either case the vessels were approached just above the internal inguinal ring (Fig. 2).

Follow-up included clinical and CDUS assessments performed 1, 3, 6 and 12 months postoperatively, then annually. Groups were compared with regard to grade of varicocele, indications for surgery (pain, growth failure of left testicle, large varicocele), incidence of recurrent/persistent varicocele, incidence of postoperative hydrocele, and postoperative catch-up growth among the affected testicles with preoperative growth failure.

Data were quoted as medians and range. Non-parametric tests were used throughout. A Chi-square (or Fisher's exact) test was used to compare frequencies between groups, and a Mann-Whitney $U$-test for non-paired continuous values (length of follow-up). A $p$ value of $\leq 0.05$ was considered significant.

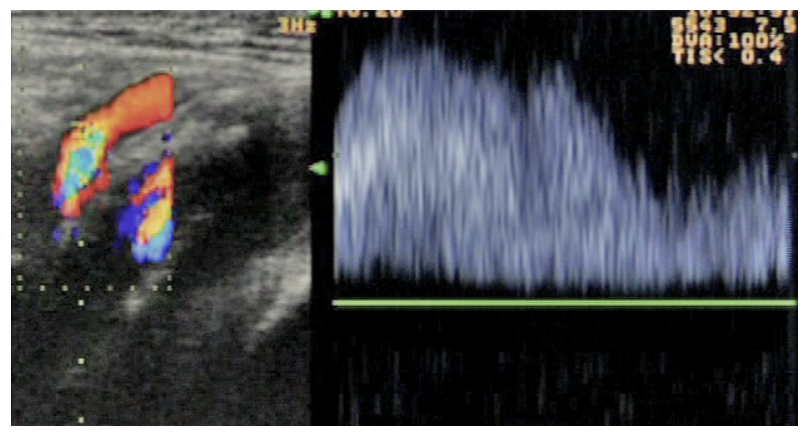

Figure 1 Deferential reflux on colour-Doppler ultrasound and related spectral analysis. 


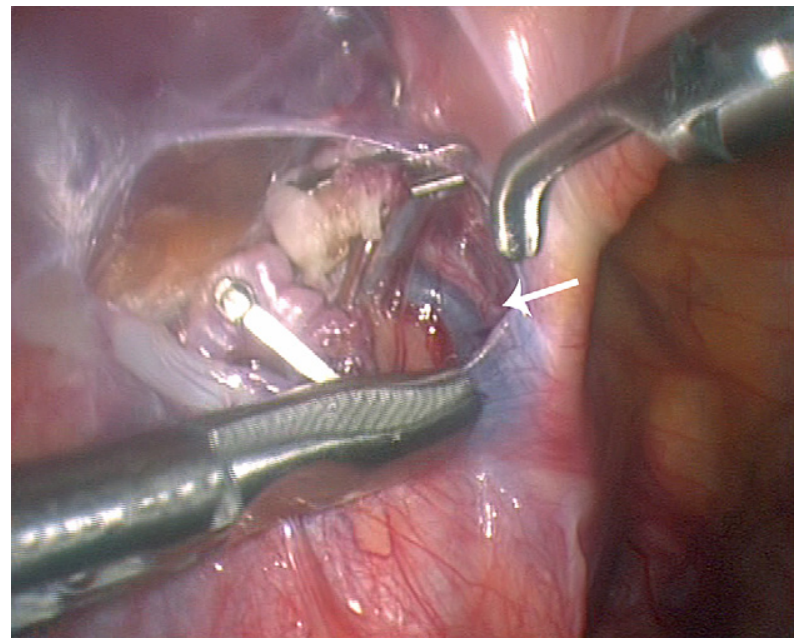

Figure 2 Intraoperative view: a peritoneal window created just cranial to the internal inguinal ring offers access to both the ISV, already sectioned in the picture, and the deferential veins, running parallel to the vas (white arrow).

\section{Results}

Fig. 3 compares the indications for surgery between the two groups. In more recent cases (group B) indication for surgery based on left testicular growth failure was more common, whereas the number of cases operated on only because of a large varicocele decreased.

Table 1 shows the surgical outcome in the two groups. Recurrence rate was slightly lower in group B but the

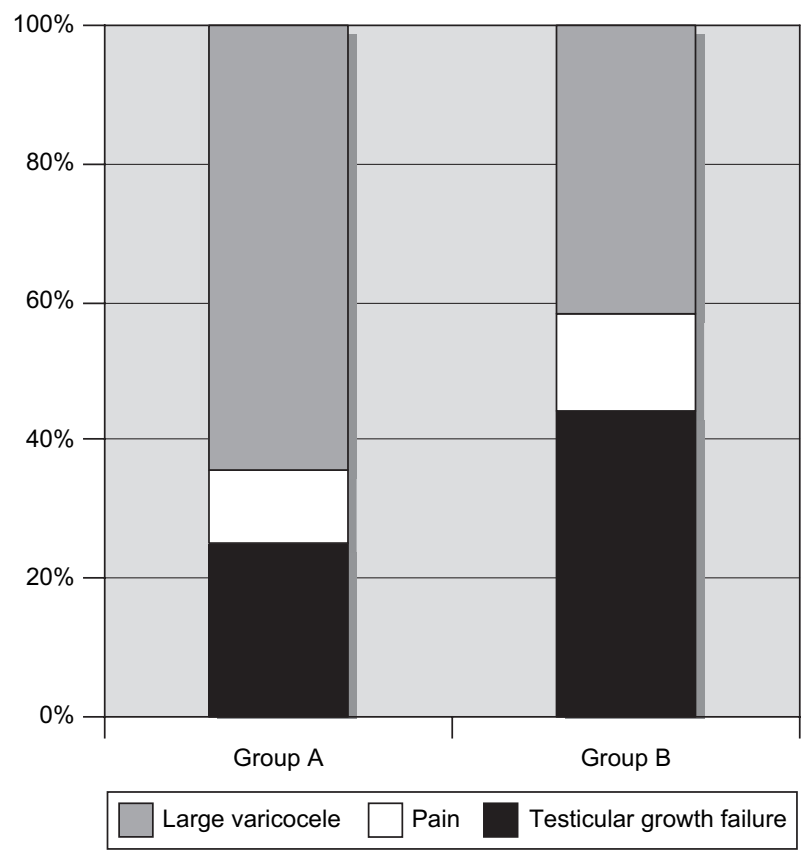

Figure 3 Prevalence of the three different indications (large varicocele, pain and growth retardation) in groups $A$ and $B$. Difference between groups was significant with regard to the number of cases with a large varicocele $(P=0.0002)$ and testicular growth failure $(P=0.001)$, but not for those presenting with pain $(P=0.1)$.
Table 1 Outcome of varicocelectomy in groups A and B

\begin{tabular}{llll}
\hline & Group A & Group B & $P$ \\
\hline $\begin{array}{l}\text { Testicular atrophy } \\
\text { Recurrent/persisting } \\
\quad \text { varicoceles }\end{array}$ & $4 / 124(3.2 \%)$ & $2 / 118(1.7 \%)$ & $>0.05$ \\
$\begin{array}{c}\text { Postoperative } \\
\text { hydroceles }\end{array}$ & $10 / 124(8.1 \%)$ & $5 / 118(4.2 \%)$ & $>0.05$ \\
$\begin{array}{c}\text { Testicular catch-up } \\
\text { growth }\end{array}$ & $23 / 31(73 \%)$ & $40 / 52(77 \%)$ & $>0.05$ \\
\hline
\end{tabular}

difference was not statistically significant. In group B, a standard Palomo procedure was performed in $103(87 \%)$ cases, whereas division of both the ISV and deferential vein was performed in the remaining $15(13 \%)$ in whom preoperative CDUS showed the presence of reflux in the deferential vein also. In no case was isolated deferential reflux observed on CDUS and no cremasteric reflux detected.

Average postoperative follow up was significantly longer $(p<0.05)$ in group A than group B patients: 7.2 years $(6.3-$ 12.1 years) vs. 3.7 (1.2-5.8 years). During follow up, postoperative hydrocele occurred in $15(6.2 \%)$ patients. It appeared in median 3 months ( 2 weeks to 11 months) after surgery and was more common in group A patients, although the difference was not statistically significant. Among group A patients, however, hydrocele formation was significantly more common $(P=0.05)$ if the vaginalis was left untouched (6 out of $41,15 \%$ ) than if it was opened during varicocelectomy (4 out of $83,4.8 \%$ ). In the latter cases, the hydrocele rate was comparable to that of group B patients $(4.8 \%$ vs. $4.2 \%)$. Postoperative hydrocele was treated surgically in all group A patients and conservatively in all group $B$ patients. Of the latter, the hydrocele has so far resolved in three out of five cases $(60 \%)$.

Finally, a comparable proportion of patients with reduced testicular volume preoperatively experienced postoperative testicular catch-up growth: group A, 73\% vs. group $B, 77 \%$.

\section{Discussion}

This study summarizes the changes in management of paediatric varicocele at our institution during the last 10 years, including a less aggressive surgical attitude and a tendency to prefer a minimally invasive approach for treatment.

Regarding the indications for surgery, nowadays we tend to operate only on cases with evidence of ongoing varicocele-related testicular damage, namely those with growth failure of the affected testis. Cayan et al. reported such a feature to occur in none of the children with varicocele aged $<11$ years, in $7 \%$ of those aged $11-$ 14 years, and in $9 \%$ of those $15-19$ years old [5]. Of these patients, only those operated on before the age of 14 years experienced postoperative testicular catch-up growth, whereas in the older ones only testicular firmness increased after surgery [5]. These data seem to support the theory that varicocele is a progressive disease and that early correction is appropriate. Diamond et al. [14], 
however, followed 41 boys with varicocele (mean age 13.7 months) for a mean of 16 months and found no change in both grade of varicocele and average testicular volume, and Greenfield et al. [15] reported postoperative catch-up growth in more than $80 \%$ of their patients undergoing varicocelectomy irrespective of age. Based on these data, both groups advocated initial non-operative management with annual follow-up controls.

A decreasing, yet relevant, proportion of our patients were operated on because of a varicocele considered large at either clinical assessment or on CDUS. Although the correlation between dimension of varicocele and testicular damage is questionable [6], diameter of the veins on preoperative US and assessment of haemodynamic characteristics (duration, velocity and refluxing volume) of the venous reflux causing the varicocele on preoperative CDUS are emerging as new parameters that may be of relevance in decision making $[12,16,17]$.

As we previously showed, CDUS may also be helpful in defining the functional anatomy of varicocele, allowing for comprehensive evaluation of the three major venous networks draining the pampiniform plexus: the ISV, the cremasteric vein(s) and the deferential vein(s). This makes possible the identification of any collateral and differentiating veins actually refluxing from those just dilated due to overflow [8]. In our experience, the ISV proved refluxing in all cases. A reflux in the deferential vein(s) was associated in some $13 \%$ of cases. In keeping with a previous venographic study by Franco et al. [18], in no patient was a cremasteric reflux detected.

Regarding treatment, microsurgical subinguinal varicocelectomy, radiological embolization and laparoscopic varicocelectomy represent the most disputed approaches. The ideal choice should be effective for any type of varicocele, have minimal morbidity, preserve optimal testicular function and be cost effective [19]. Comparison of costs is very difficult, as costs seem to vary widely among countries, and depend on the caseload of the centre and on whether the equipment is dedicated only to the treatment of varicocele or used also for other conditions [20,21]. Final testicular function is a questionable end-point in children as well, as it is difficult to differentiate postoperative from spontaneous improvement. Even if we just consider catch-up growth, which occurred in some $75 \%$ of our patients irrespective of the surgical procedure, this might be more affected by age at surgery than by surgical technique [5]. Therefore, postoperative varicocele recurrence/persistence and hydrocele formation represent the two outcome measures that allow for the most consistent comparison among approaches.

Open subinguinal microsurgical varicocelectomy, allowing simultaneous exposure of all three major venous systems forming the pampiniform plexus, seems the most flexible approach. With the aid of the operating microscope and intraoperative Doppler, this technique allows both an artery- and lymphatic-sparing varicocelectomy. Varicocele recurrence/persistence and hydrocele rates can be as low as $0 \%$ and $2 \%$, respectively [22]. The major drawback is the need for microsurgical skills. We performed subinguinal varicocelectomy with the aid of loupes (3.5× magnification), and our recurrence/persistence and hydrocele rates were $3.2 \%$ and $8.1 \%$, respectively. Among patients treated by this approach, postoperative hydrocele was significantly less common if the testicular vaginalis was resected and everted at surgery than if it was left untouched $(4.8 \%$ and $15 \%$, respectively).

Radiological treatment can be performed in either a retrograde or antegrade fashion. The former is associated with a $10-15 \%$ rate of technical failure in children, which make it impractical [21]. Antegrade sclerotherapy has the drawback of requiring a combination of surgical skills to dissect a vein in the scrotum for cannulation, and radiological equipment. Being an entirely endovascular procedure, it does not carry any risk of hydrocele formation. Recurrence/persistence rate varies between $7 \%$ and $20 \%$ $[20,23]$, a possible reason for recurrence being that this approach only addresses a possible reflux in the ISV.

Laparoscopic and retroperitoneoscopic varicocelectomy have gained popularity in the last decade. The retroperitoneoscopic approach is theoretically more physiological, but being also more troublesome is less widely adopted. Irrespective of the approach, the procedure is a Palomo high ligature of the ISV. This can either be performed as artery sparing or not. Ligature of the artery does not increase the risk of testicular atrophy, does reduce the risk of varicocele recurrence, and does increase the risk of hydrocele formation [24,25].

In order to increase the success of surgery, we have introduced some technical refinements to our laparoscopic procedure. To minimize the risk of leaving behind any refluxing collaterals, we still consider the interruption of the ISV crucial, but we also think it important to approach the veins as close as possible to the internal inguinal ring, since at this level all the vessels converge. The optical magnification of the laparoscope and the broad anatomical view through the peritoneum allow for precise identification of all the dilated veins, as well as for sparing the lymphatic vessels in order to also prevent postoperative hydrocele [26]. Use of a vital dye enhancing the lymphatic vessels after injection in the scrotum close to the tunica vaginalis has been suggested to make lymphatic sparing easier [27]. We rather believe it to be enough to perform meticulous surgery with the aid of magnification and minimize any manipulation of the retroperitoneal fat on which the lymphatic vessels lie. With this method we achieved a reasonably low hydrocele rate $(4.2 \%)$ for a non-arterysparing technique [25].

The major innovation of our approach consists of a selective ligature and interruption even of the deferential vein when this proves refluxing on preoperative CDUS assessment. In our opinion, this represents a rigorous standard that takes into account the complex vascular anatomy of varicocele. Dudai et al. [28] previously described a modified laparoscopic procedure including systematic interruption of both the ISV and the inferior epigastric vessels. Although the principle is similar, the two techniques are very different in that our approach is selective and not systematic, and interruption of the inferior epigastric vessels actually addresses only a possible cremasteric reflux whose role in the onset of varicocele may indeed be questioned based on both our experience and that of Franco et al. [18].

Another important trend shown in our study is towards a more conservative management of postoperative 
hydrocele. This is consistent with a multi-institutional study by Esposito et al. [25], concluding that non-invasive procedures, such as scrotal punctures and clinical observation, can induce total hydrocele regression in more than $80 \%$ of cases.

We acknowledge that the current study has several limitations, due to its retrospective design involving comparison of two groups operated on consecutively. In 19 (15\%) of group A patients the testicular volume was assessed only by an orchidometer. This is a reliable method but less accurate than US, used in all the other patients, to assess testicular volume differential [29]. The shorter average follow up in group $B$ might have contributed to the lower hydrocele rate in this group. Recent reports documented cases of hydrocele occurring up to 3 years after varicocelectomy [30]. In this respect, however, in our experience as well as that of Esposito et al. [25], hydroceles generally occurred in the first months after varicocelectomy, and average follow up was anyway longer than 3 years in both our groups. Finally, the current study concerns only two of the three major treatment modalities discussed. In spite of these limitations, we think our experience should still be of use by indicating that success of treatment depends more on accurate preoperative assessment and treatment selection than on the surgical technique itself.

In conclusion, this study shows that the indications for treatment of adolescent varicocele remain quite controversial. Varicocele can, however, be successfully treated in the majority of cases by either a laparoscopic or a subinguinal approach, provided that either technique is performed carefully in order not to miss any possible refluxing collateral. With this purpose in mind, preoperative CDUS allows for a comprehensive evaluation of the vascular anatomy of varicocele and aids in the selection of the most appropriate technique. If surgery is performed for testicular growth failure, most of the patients can be expected to experience postoperative testicular catch-up growth.

\section{Conflict of interest}

There is no conflict of interest to disclose. This study was not supported by any funding source. Institutional review board approval was not required.

\section{References}

[1] Jarow JP, Coburn M, Sigman M. Incidence of varicoceles in men with primary and secondary infertility. Urology 1996;47:73.

[2] Akbay E, Cayan S, Doruk E, Duce MN, Bozlu M. The prevalence of varicocele and varicocele related testicular atrophy in Turkish children and adolescents. BJU Int 2000;86: 490-3.

[3] Meacham RB, Townsend RR, Rademacher D, Drose JA. The incidence of varicoceles in the general population when evaluated by physical examination, gray scale sonography and color Doppler sonography. J Urol 1994;151:1535-8.

[4] Lund L, Tang YC, Roebuck D, Lee KH, Liu K, Yeung CK. Testicular catch-up growth after varicocele correction in adolescents. Pediatr Surg Int 1999;15:134-7.

[5] Cayan S, Akbay E, Bozlu M, Doruk E, Erdem E, Acar D, et al. The effect of varicocele repair on testicular volume in children and adolescents with varicocele. J Urol 2002; 168:731-4.

[6] Diamond DA, Zurakowski D, Bauer SB, Borer JG, Peters CA, Cilento Jr BG, et al. Relationship of varicocele grade and testicular hypotrophy to semen parameters in adolescents. J Urol 2007;178:1584-8.

[7] Coolsaet BL. The varicocele syndrome: venography determining the optimal level for surgical management. J Urol 1980; 124:833-9.

[8] Cimador M, Di Pace MR, Peritore $M$, Sergio $M$, Castagnetti $M$, De Grazia E. The role of color-doppler ultrasound in determining the proper surgical approach to the management of varicocele in children and adolescents. BJU Int 2006;97: 1291-7.

[9] Cimador M, Castagnetti M, Ajovalasit V, Libri M, Bertozzi M, De Grazia E. Sub-inguinal interruption of dilated veins in adolescent varicocele: should it be considered a gold standard technique? Minerva Pediatr 2003;55:599-605.

[10] Cimador M, Di Pace MR, Castagnetti M, Catalano P, Sergio M, De Grazia E. Comprehensive laparoscopic approach to paediatric varicocele based on preoperative colour-doppler ultrasound assessment. Surg Endosc 2007; doi:10.1007/s00464-007-9464-9.

[11] Dubin L, Amelar RD. Varicocele size and results of varicocelec tomy in selected subfertile men with varicocele. Fertil Steril 1970;21:606-9.

[12] Hussein AF. The role of color Doppler ultrasound in prediction of the outcome of microsurgical subinguinal varicocelectomy. J Urol 2006;176:2141-5.

[13] Rodriguez WC, Rodriguez DD, Fortuno RF. The operative treatment of hydrocele: a comparison of four basic techniques. J Urol 1981;125:804-6.

[14] Diamond DA, Zurakowski D, Atala A, Bauer SB, Borer JG, Cilento BG, et al. Is adolescent varicocele a progressive disease process? J Urol 2004;172:1746-8.

[15] Greenfield SP, Seville P, Wan J. Experience with varicocele in children and young adults. J Urol 2002;168:1684-8.

[16] Nagar H, Mabjeesh NJ. Decision-making in pediatric varicocele surgery: use of color Doppler ultrasound. Pediatr Surg Int 2000;15:75-6.

[17] Tanrikut C, Goldstein M. Varicocele: size does matter. J Urol 2006;176:1912-3.

[18] Franco G, lori F, de Dominicis C, Dal Forno S, Mander A, Laurenti $C$. Challenging the role of cremasteric reflux in the pathogenesis of varicocele using a new venographic approach. J Urol 1999;161:117-21.

[19] Goldstein M. Adolescent varicocele. J Urol 1995;153:484-5.

[20] Mazzoni G, Fiocca G, Minucci S, Pieri S, Paolicelli D, Morucci $M$, et al. Varicocele: a multidisciplinary approach in children and adolescents. J Urol 1999;162:1755-8.

[21] Beutner S, May M, Hoschke B, Helke C, Lein M, Roigas J, et al. Treatment of varicocele with reference to age: a retrospective comparison of three minimally invasive procedures. Surg Endosc 2007;21:61-5.

[22] Schiff J, Kelly C, Goldstein M, Schelegel P, Poppas D. Managing varicoceles in children: results with microsurgical varicocelectomy. BJU Int 2005;95:399-402.

[23] Zupa P, Mayr J, Hollwarth E. Antegrade scrotal sclerotherapy for treating primary varicocele in children. BJU Int 2006;97: 809-12.

[24] Sun N, Cheung TT, Kong PL, Chan KL, Tam PKH. Varicocele: laparoscopic clipping and color Doppler follow-up. J Pediar Surg 2001;36:1704-7.

[25] Esposito C, Valla JS, Najmaldin A, Shier F, Mattioli G, Savanelli A, et al. Incidence and management of hydrocele following varicocele surgery in children. J Urol 2004;171:1271-3.

[26] Kocvara R, Dvorácek J, Sedlácek J, Díte Z, Novák K. Lymphatic sparing laparoscopic varicocelectomy: a microsurgical repair. J Urol 2005;173:1751-4. 
[27] Oswald J, Korner I, Riccabona M. The use of isosulphan blue to identify lymphatic vessels in high retroperitoneal ligation of adolescent varicocele-avoiding postoperative hydrocele. BJU Int 2001;87:502-4.

[28] Dudai M, Sayfan J, Mesholam J, Sperber Y. Laparoscopic simultaneous ligation of internal and external spermatic veins for varicocele. J Urol 1995;153:704-5.
[29] Diamond DA, Paltiel HJ, DiCanzio J, Zurakowski D, Bauer SB, Atala A, et al. Comparative assessment of pediatric testicular volume: orchidometer versus ultrasound. J Urol 2000;164:1111-4.

[30] Misseri R, Gershbein AB, Horowitz M, Glassberg KI. The adolescent varicocele. II. the incidence of hydrocele and delayed recurrent varicocele after varicocelectomy in a long-term follow-up. BJU Int 2001;87:494-8. 